\title{
MEETING ETHICAL DEMANDS IN A MULTI-AGENCY PROJECT
}

\author{
Joe Thomas \& Andy Bissett \\ School of Computing \& Management Sciences, \\ Sheffield Hallam University, England S1 1WB
}

\begin{abstract}
Various health service and voluntary sector agencies dealing with drug misusers in a large city in the North of England have set up a task group to co-ordinate their assessment and referral process. An IT solution to their needs was believed to be desirable but raises serious issues concerning confidentiality, and the suitability of an IT solution to the particular information problem. The approach is informed by Social Informatics and utilises Soft Systems Methodology within the framework of Joint Application Development.
\end{abstract}

\section{INTRODUCTION}

This paper describes a piece of action research undertaken as an MSc dissertation project between June and September 2000 [Thomas, 2000]. The project involved the provision of an IT solution for a task group of health service and voluntary sector agencies involved in providing services to drug misusers in a large city in the North of England.

The task group was charged with co-ordinating the various agencies assessment and referral process. This was considered to be desirable as many of the clients of these services are involved with several agencies, either simultaneously or serially, and each agency assessed their clients separately, and may then refer them on to other agencies to be assessed again. The nature of this process was found to be repetitive and contributed to potential clients failing to seek help from the agencies (there is some anecdotal evidence that a small minority of drug misusers upon being convicted of an offence would rather accept a custodial sentence than a suspended sentence on condition that they use the agencies' services). Although each agency has a different role (counselling, medical care, residential rehabilitation, advisory etc.) the core of the information they collect is essentially similar. The task group was set up to work towards a common assessment tool to allow information to be gathered at the clients entry point to the services, and to be passed on and added to as they progressed through whichever agencies were appropriate for them.

In addition to streamlining the process to provide a better service to the drug misusers, the task group wished to co-ordinate the process in order to allow a centralised collection of information in order to produce accurate statistics regarding the nature and size of their client base to assist with planning and resourcing the services more efficiently. However the need to produce summary information could not be allowed to compromise client confidentiality, so, although the records must be identifiable (to prevent double-counting) they could not identify individuals.

The ethical dimension of this problem arises mainly around issues of confidentiality, as each agency has its own agreements and understandings with its clients. Often clients divulge information to one agency that they do not wish to be passed on to any other agencies they become involved with, even if they are referred. Legislation such as the Data Protection Act had proven to be a stumbling block for similar projects undertaken by DATs (Drug Action Team - a local co-ordinating body) in other parts of the country, and regulations about the gathering and confidentiality of medical records created a divide between health service and voluntary sector agencies. In addition to this the clients' own agendas and permissions regarding the sharing of information needed to be addressed.

Also in need of consideration was how the assessment and referral process would integrate with their existing administrative duties. The health service agencies have particular difficulties in this area as they already have statutory paperwork requirements. In addition they do not collect all the information at the same time and it is performed under a number of different roles. The example given was that the reception staff would collect personal details like name, address, date of birth etc., and a nurse would complete separate paperwork for risk assessment, substance misuse history and so forth.

The approach adopted by the researcher was informed by the field of Social Informatics [Kling, 1996; 1998; 2000] and used Checkland's [Checkland, 1981; Checkland \& Scholes, 1990; Checkland \& Holwell 1998] Soft System Methodology as an analytical tool, and Joint Application Development [Wood \& Silver, 1995] as a framework to structure contact with the agencies.

This paper will consider the project as a piece of action research, before discussing the epistemology of systemic thinking and Social Informatics. There follows a discussion of the methodology applied and the successes and failures of this methodology in an ethically charged and complex problem area. 


\section{THE PROJECT AS ACTION RESEARCH}

\section{Action Research}

The research project was essentially a piece of action research, as defined by Checkland and Holwell [Checkland \& Holwell, 1998]. The epistemological basis on which Checkland and Holwell build their definition of action research is defined as interpretivist, as opposed to positivist, or functionalist.

Their account of action research requires the researcher to adopt this interpretivist approach, and accepts the common notion that action research will entail the researcher becoming actively involved with the problem situation and more importantly, the people in the situation. Emphasis is placed on understanding the social practice of the people involved, and the role that the problem situation plays for them, how it is integrated with their other processes and practices.

Action research was selected as the mode of enquiry for the project as it provides useful concepts for understanding of the complex social situation existing between the members of the task group. The interpretivist approach offered by action research represents a less problematic understanding of the problem domain than a positivist approach could. The focus of the project is the discovery of the requirements for a proposed system, and an analysis of the feasibility of implementing these requirements, not the technical problem of designing and implementing the system, although obviously the two are not easily separable. The building of a prototype, for example, by necessity addresses design issues, but its primary function and importance is as a confirmation that the requirements are being addressed.

\section{Epistemology - Systemic Thinking and Social Informatics}

Soft Systems Methodology (SSM) was selected to provide an appreciation of the 'culture' of the group and its member organisations, as soft systems thinking is considered to be particularly effective in analysing complex organisational problems. Social Informatics was selected in combination with systemic thinking to provide a strong scepticism regarding the assumptions the group had already made, that the answer to their information systems problem was an IT solution. As Kling states [1999] of the critical orientation of Social Informatics, "The critical orientation refers to examining information technologies from perspectives that do not automatically and 'uncritically' adopt the goals and beliefs of the groups that commission, design, or implement specific information technologies." Social Informatics also incorporates concepts that are familiar from the discussion of action research and SSM. The holistic approach of Social Informatics seems to fit comfortably with the action research (and SSM) ethic of considering the social and organisational factors surrounding an Information System problem in as great a depth as any proposed Information Technology solution.

\section{METHODOLOGY: A COMBINATION OF JAD AND SSM}

\section{Joint Application Development and Soft Systems Methodology}

The research was conducted along the lines of an informal Joint Application Development (JAD) process, [Wood and Silver, 1995]. The JAD process was adopted for a number of reasons, the foremost being the assembly of the task group, in effect this group was the JAD group as it consisted of managers from all the relevant bodies. The authority existed within the group representatives to instigate and implement any findings from the project research, especially with the involvement of the area co-ordinator.

The application of this process was somewhat problematic. The literanure refers to the JAD process as involving departments within a single organisation, and although the group is relatively cohesive; the fact that they are separate, or disparate, entities can lead to a lack of unified direction. However, the group does have a common interest: the best possible provision of services to drug users in the area, although they may not always agree on what this means, and all will naturally focus on the services their own agencies provides.

The researcher filled the role of scribe and facilitator. The major issue with this selection is one of authority and the groups perception of the researchers role - It became apparent there were different perceptions of the role the author should play. The group felt they required a research student to act as an IT consultant, and to take their requirements away and produce a database. The action research and epistemological approach selected challenged this definition, and required a deeper investigation into their requirements and culture. As a research student (and lacking an executive sponsor to appeal to for authority), the facilitator did not have the authority to lead the group, in the way Wood and Silver would perhaps advise. However, the group are quite informal and friendly, and were keen to receive advice, there was no point at which major issues of authority arose. Regardless of positive experiences in this study it remains a point worthy of consideration in future research. 


\section{The Methodology in Action}

An issue was raised about the paper records kept by most of the agencies. It was felt that a system that required a worker to check computer records and paper records would actually be more time consuming and less efficient than the existing practice of simply locating a client file in a filing cabinet. In addition, the usefulness of the paper form that had been prepared as a template for a database was not as immediately clear to the author as it appeared to be to the task group. The form contains several very long text fields in the later pages, and these was causing concern amongst the workers that it could be labour intensive, especially for those with slow typing speeds. Not all the agencies have administrative staff to complete these duties, and those that do would still require a worker to complete a paper form to pass on for data entry. Long text fields would also be of very limited use in producing statistics and searches for possible entries would need to be impractically exhaustive.

The issues of administration time and cost were also raised. The interviews conducted with the agencies had exposed differences in the methods of information gathering. Doctors at the Primary Care Clinic, already used a specially designed software package, and input the information interactively with his clients, during consultations. Other agencies interviewed their clients in meeting rooms or in the clients' homes, in areas without access to computers. There was a general consensus amongst the voluntary sector agencies that the use of computers (desktop or laptop) in meetings could be distracting or intrusive, and in some cases may represent a security risk, in addition to representing a large additional budgetary consideration. The representative from the Primary Care Clinic's suggestion that the other agencies adopt the health service practice of using PCs interactively with their clients was not considered to be wholly practical for the voluntary sector agencies. A Local Drugs Project manager, in an interview with the author, had suggested that the practice was more viable for health service agencies due to the difference in the clients' expectations of how health service and voluntary sector agencies' records would be kept. Clients would expect medical records to be scrupulously recorded and available across the health service, but would view the same practice with extreme suspicion if conducted by counselling and residential care agencies, where care is based more heavily on personal relationships and trust. Perceptions (by the clients) of how the information would be used could also be problematic, as the recording of information electronically could create uncertainty as to the scope of access to it. Assurances that access to a stand-alone system was purely within the agency that recorded it, and that information would only be transferred at referral with the client's consent was not, in his opinion, necessarily sufficient to allay this concern. There are obviously ethical issues involved here. If the voluntary service agencies were unwilling to complete computer records in the presence of the client (because this could undermine the trust that the information is confidential), is it ethical for them to enter the records when the client is not present, and perhaps not aware?

The use of SSM to analyse the problem situation requires a root definition drawn from the CATWOE mnemonic, which draws attention to the requirements in a slightly different manner, in the below example.

C - Customers: the agencies involved in providing services to drug users in the local area, and indirectly their clients

A - Actors: the research student and the agencies represented in the task group T - Transformation: information about clients recorded in varying formats but not shared or analysed $\rightarrow$ information about clients recorded in a unified format, shared where appropriate and analysed at an agency and regional level

W - Weltanschauung: Social Informatics used to address Systemic Thinking. Sharing and analysing information will result in a better provision of services.

O - Owners: the agencies involved in the task group and agencies not involved in the task group but involved in providing services to drug users

E - Environmental Client confidentiality, budget and time available for

Constraints the MSc project, expertise of the research student, the existing IT in place at the agencies, the agencies working practices, the attitudes of the clients

From this mnemonic a root definition can be drawn as follows:

"A system to record, share and analyse client information presented in a uniform format across the agencies that address issues of drug misuse in the local area in order to provide a better service to the clients."

The mnemonic also draws attention to a previously un-stated assumption of the project: that the recording, sharing and analysis of client records will enable the agencies to provide a 'better' service. The assumption seems sound based on the information provided by the group, but is extremely subjective. It is not difficult to imagine that some clients enjoy the anonymity that a system that does not share information provides, as this enables them to represent themselves as they wish each time they come into contact with a new service. For clients who 
wish to misrepresent themselves, perhaps to manipulate the services in their favour, sharing of information is not beneficial at all, from their perspective. Issues of trust have been raised previously, and the possibility that clients may perceive wider access to their personal details as intrusive, even under the controlled circumstances the system would allow, is very real, and could form a serious barrier to the personal bond between worker and client.

An example of one of the SSM rich pictures prepared during the course of the project appears below.

\section{Complex Organisational Rich Picture: Requirements \& Issues}

$\begin{array}{ll}\text { "we need to formulate a city } & \text { "we have our own } \\ \text { action plan, we need figures, } & \text { paperwork - we still need to } \\ \text { statistics etc" } & \text { re-assess clients" }\end{array}$

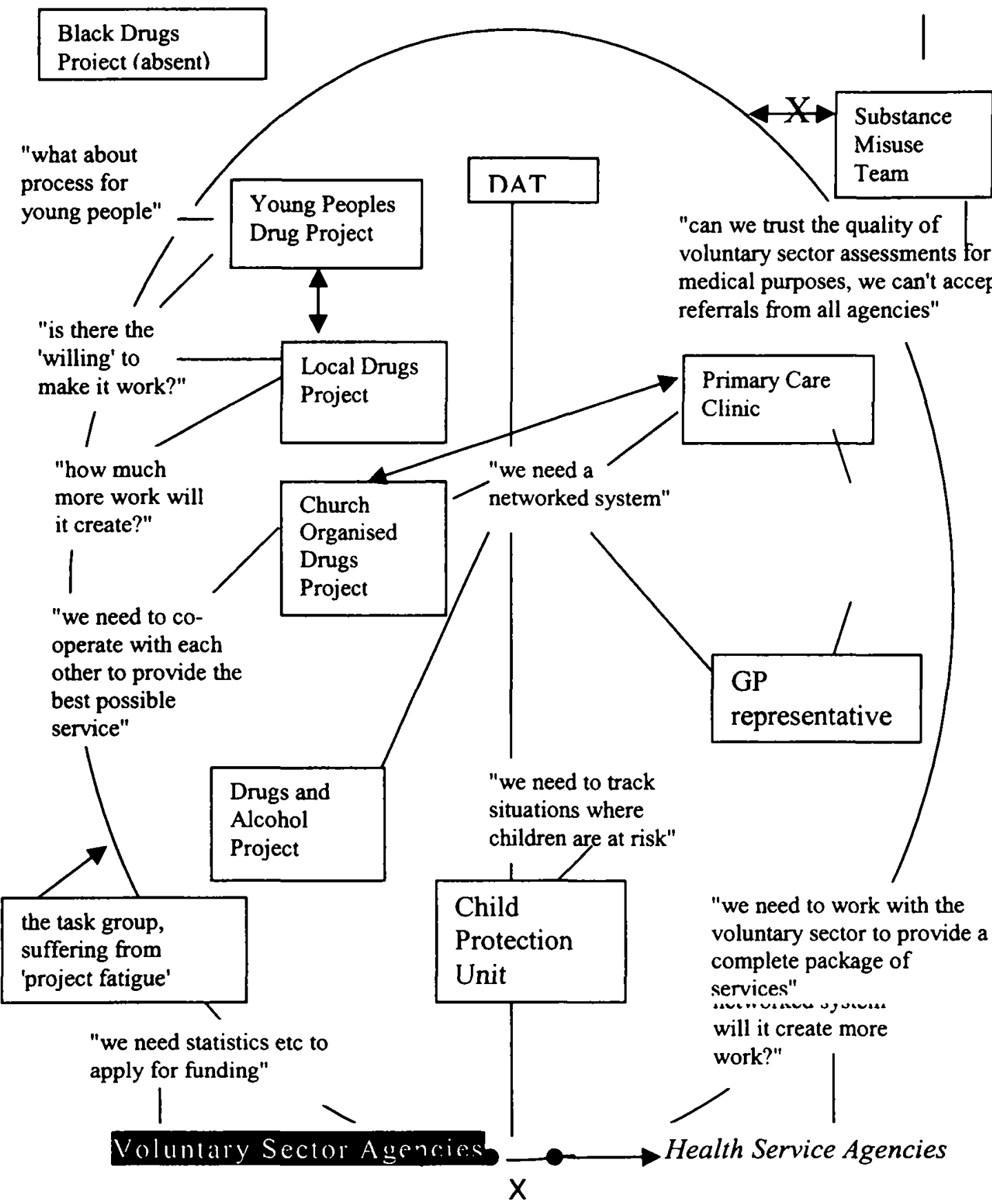


The research stage yielded a great deal of insight into the very different contexts in which a system would be used. Most of the voluntary sector agencies perceive their work as meeting clients and forming a personal relationship (this is an over-simplification, intended to serve as an illustration of the culture) with the record keeping serving as a subsidiary function. The health service by practice, tradition and statute requires scrupulous and comprehensive records without which it cannot function. Whilst the practices of the two are becoming more closely intertwined this is still a significant distinction. The voluntary sector agencies tend towards a higher number of workers per PC, and are not in a position to consider IT central to the services they provide. This is especially significant when considering the theories behind Social Informatics, as this environment (or social context) is far less conducive to efficient use of an IT system.

The session itself, phase 4 of the JAD process, took place at the task group meeting 26 July 2000 . The key elements of the session were adhered to:

- A discussion of the assumptions that had been made by the various agencies, and how these differed from each other, e.g. the difference in practice between health service and voluntary sector agencies in use of PCs

- A discussion of precisely what data was required. Interviews had led to the proposal not all of the data from the assessment form be included, but that whatever data was entered would need to be updated and stored, a recording of assessment information only had not been considered viable by interviewees or by the author.

- A discussion of the 'business processes'. This overlapped heavily with the discussion of data requirements. The research had attempted to place the process the group wanted to automate (record keeping for assessments) in the context of the wider processes (record keeping about the client)

The session yielded fruitful discussion, but also caused concern that the group had agreed to all the general proposals made by the researcher. As they were receiving 'free' advice a possible bias towards acceptance of the proposals, as this would result in further possible benefits without costs, may have been present, and prevented free expression of further, or more complex requirements. As a mitigating factor, the viability of a networked database (which had been the groups original aim) had been dismissed due to the project timeframe and budget, and it was proposed that an Access database be implemented, either for use in maintaining and sharing records or to demonstrate the requirements a networked database would have. The group made provisional agreements (that the prototype should be developed) without committing themselves to using it.

One of the agencies involved in the task group is currently piloting a 'beta version' of the database, with a view to rolling out the application to the other agencies once any outstanding issues have been identified and resolved. Technologically, the application falls deliberately short of the distributed database the group initially believed they required; this compromise allows them to meet their confidentiality requirements whilst implementing a relatively low cost system.

\section{CONCLUSION}

As is a common problem with action research, the interpretivist approach adopted makes it difficult to gauge what conclusions can be drawn that are not inextricably linked with the context of the particular area of study. It seems clear that Checkland and Holwell's prescribed 'immersion' in the problem area can be a poor definition of the boundaries of a project and 'scope creep' can easily ensue. However, given that software development is an area in which project failures or high cost maintenance problems are often attributed to an inadequate analysis of what is required at the initial stages, an approach which attempts to rigorously scrutinise the users stated needs and the environment in which the system will be used seems appropriate.

It appears that the deviation from the JAD process to concentrate more heavily on gathering and clarifying requirements and generating options using SSM resulted in a richer understanding of a complex and ethically charged situation, providing a basis for a system that took a more balanced consideration of the needs of its various stakeholders than an early acceptance of the group's stated requirements.

Taking ethical needs into account may result in a proposed solution that is not so technically optimal as the technologically driven discourse of IT systems development might suggest.

\section{REFERENCES}

Checkland, P (1981) Systems Thinking, Systems Practice John Wiley \& Sons Ltd

Checkland, P \& Holwell, S (1998) Information, Systems and Information Systems John Wiley \& Sons Ltd

Checkland, P \& Scholes, J (1990) Soft Systems Methodology in Action John Wiley \& Sons Ltd

Kling, R (1999) 'What is Social Informatics and Why Does it Matter?' D-Lib Magazine January 1999, 5 (1) accessed at http://www.dlib.org/dlib/january99/kling/01kling.html, 27 June 2000 
Kling, R, Crawford, H, Rosenbaum, H, Sawyer, S \& Weisband, S (2000)Learning from Social Informatics: Information and Communication Technologies in Human Contexts (Draft) Centre for Social Informatics, University of Indiana accessed at http://www/slis.indiana.edu/kling

Thomas, J (2000) Feasibility Study for a Multi-Agency Client Assessment and Referral Database, Sheffield Hallam University

Wood, J \& Silver, D (1995) Joint Application Development ( $2^{\text {nd }}$ edition), John Wiley \& Sons Inc 